\title{
INSTABILITY OF VISCOUS FLOWS OVER A SHRINKING SHEET
}

\author{
BY \\ M. MIKLAVČIČ \\ Department of Mathematics, Michigan State University, East Lansing, Michigan 48824
}

\begin{abstract}
We prove instability of a part of a branch of viscous incompressible fluid flows induced by a shrinking sheet. These flows are exact solutions of the Navier-Stokes equation.
\end{abstract}

1. Introduction. The main purpose of this paper is to prove instability of an exact solution of Navier-Stokes equations for an incompressible fluid. There are many stability results available [2. For example, in bounded domains, the 0 solution is stable. This is basically due to the fact that $-\triangle$ has a spectrum in $[\varepsilon, \infty)$ for some $\varepsilon>0$. In unbounded domains $\varepsilon=0$ but use of weighted spaces can sometimes push the spectrum away from the zero, and stability can be proved for small Reynolds numbers [4. To show instability of a viscous flow to arbitrarily small perturbations, one basically needs to show existence of an unstable eigenvalue corresponding to the linearization around the flow. This was done numerically many times, but to prove the existence of one is a different matter due to the complicated nature of the Navier-Stokes equations. Fairly recently [6], a number of exact solutions to the Navier-Stokes equations was discovered. For a branch of those solutions, describing a flow of an incompressible fluid over a shrinking plate with suction, both eigenfunctions corresponding to 0 eigenvalue of the linearization are presented explicitly, and we prove that the eigenvalue crosses an imaginary axis transversally.

Let $(u, w)$ be velocity components of an incompressible fluid in the Cartesian directions $(x, z)$ respectively. The time-dependent Navier-Stokes equations are

$$
\begin{gathered}
u_{x}+w_{z}=0 \\
u_{t}+u u_{x}+w u_{z}=-p_{x} / \rho+\nu \nabla^{2} u, \\
w_{t}+u w_{x}+w w_{z}=-p_{z} / \rho+\nu \nabla^{2} w .
\end{gathered}
$$

Here $p$ is the pressure, $\rho$ is the density and $\nu$ is the kinematic viscosity. The boundary conditions on the sheet at $z=0$ are

$$
u=-a x, \quad w=-W
$$

Received August 8, 2012.

2010 Mathematics Subject Classification. Primary 35Q30, 76E09, 35K58, 76D03, 76E25.

E-mail address: milan@math.msu.edu 
where $a>0$ is the shrinking constant and $W$ is the suction velocity. Far from the sheet, the fluid has no lateral velocities and the pressure is uniform. We will study solutions of equations (1.1.1.3) that are in the form

$$
u=a x f_{\eta}(\eta, \tau), \quad w=-\sqrt{a \nu} f(\eta, \tau)
$$

where $\eta=z \sqrt{a / \nu}$ and $\tau=$ at. Equation (1.1) is automatically satisfied. Pressure $p=p(z, t)$ can be obtained from equation (1.3). Equation (1.2) reduces to the following $\mathrm{PDE}$

$$
f_{\eta \tau}=f_{\eta \eta \eta}+f f_{\eta \eta}-f_{\eta}^{2}
$$

and the boundary conditions are

$$
f(0, \tau)=\frac{W}{\sqrt{a \nu}} \equiv s, \quad f_{\eta}(0, \tau)=-1, \quad f_{\eta}(\infty, \tau)=0 .
$$

Equations (1.5, 1.6) have stationary solutions [6]

$$
f_{0}(\eta)=C+\frac{1}{C} e^{-C \eta} \quad \text { where } \quad C=\frac{s \pm \sqrt{s^{2}-4}}{2} .
$$

Observe that suction needs to be big enough $(s \geq 2)$ for these solutions to exist and that we get two solutions at each $s>2$ : one with $C>1$ and one with $C<1$. It will be shown that the branch $0<C<1$ is unstable. We will prove this for $C$ close to 1 in Sections 2 and 3 and show it numerically in Section 4. For the precise meaning of instability, see Theorem 2.2 below.

Equation (1.5) was studied in [7] for a flow over a stretching sheet. The stationary solution in this case is known as Crane's solution and was shown to be stable to small perturbations. We show here numerically that (1.7) is stable for small perturbations when $C>1$ and prove it for $C>\sqrt{10}$. However, allowed small perturbations of $u, w$ belong to a rather restricted set; see equations (1.4, 2.1). Instability established in such a restricted set will of course hold in a larger set too.

2. Semilinear parabolic equation. Let us look for solutions of equations (1.5) 1.6) that are in the form

$$
f(\eta, \tau)=C+\frac{1}{C} e^{-C \eta}+\int_{0}^{\eta} e^{-C r / 2} \psi(r, \tau) d r
$$

where $C>0$ and $s=C+1 / C$. The above weight $e^{-C r / 2}$ is used to initially separate the point spectrum from the continuous spectrum and is discussed in Section 5. The perturbation $\psi$ has to satisfy

subject to

$$
\begin{aligned}
\psi_{\tau}-\psi_{\eta \eta}+ & \frac{C^{2}}{4} \psi-\frac{e^{-C \eta}}{C} \psi_{\eta}-\frac{3 e^{-C \eta}}{2} \psi-C e^{-C \eta / 2} \int_{0}^{\eta} e^{-C r / 2} \psi(r, \tau) d r \\
& =\left(\psi_{\eta}-C \psi / 2\right) \int_{0}^{\eta} e^{-C r / 2} \psi(r, \tau) d r-e^{-C \eta / 2} \psi^{2}
\end{aligned}
$$

$$
\psi(0, \tau)=0, \quad \psi(\infty, \tau)=0, \quad \psi(\eta, 0) \text { to be given. }
$$

We will interpret (2.2, 2.3) as an abstract evolution equation, see [1] or [5],

$$
\psi^{\prime}+A \psi=h(\psi)
$$


in a Hilbert space $H=L^{2}(0, \infty)$ with the usual inner product $(\cdot, \cdot)$.

Let $V=W_{0}^{1}(0, \infty)$. Define $F: V \times V \rightarrow \mathbb{C}$ by

$$
F(u, v)=\int_{0}^{\infty}\left(u^{\prime} \bar{v}^{\prime}+\frac{C^{2}}{4} u \bar{v}-\frac{e^{-C \eta}}{C} u^{\prime} \bar{v}-\frac{3 e^{-C \eta}}{2} u \bar{v}-C e^{-C \eta / 2} \bar{v} \int_{0}^{\eta} e^{-C r / 2} u(r) d r\right) d \eta .
$$

Note that for $u \in V$

$$
\operatorname{Re} F(u, u)=\int_{0}^{\infty}\left(\left|u^{\prime}\right|^{2}+\left(\frac{C^{2}}{4}-2 e^{-C \eta}\right)|u|^{2}\right) d \eta-\frac{C}{2}\left|\int_{0}^{\infty} e^{-C r / 2} u(r) d r\right|^{2} .
$$

Using the simplest bounds one obtains

$$
\operatorname{Re} F(u, u) \geq \int_{0}^{\infty}\left|u^{\prime}\right|^{2}+\frac{C^{2}-10}{4} \int_{0}^{\infty}|u|^{2}
$$

and hence $F$ is a sectorial form (see p. 80 in [5]). This form defines a linear operator $A$ by

$$
F(u, v)=(A u, v) \quad \text { for } v \in V
$$

It is easy to see that

$$
A u=-u^{\prime \prime}+\frac{C^{2}}{4} u-\frac{e^{-C \eta}}{C} u^{\prime}-\frac{3 e^{-C \eta}}{2} u-C e^{-C \eta / 2} \int_{0}^{\eta} e^{-C r / 2} u(r) d r
$$

for $u \in D(A)=W^{2}(0, \infty) \cap W_{0}^{1}(0, \infty)$ - which is what we want in (2.4). Inequality (2.7) implies that $\sigma(A)$, the spectrum of $A$, satisfies

$$
\operatorname{Re} \sigma(A) \geq \frac{C^{2}-10}{4} .
$$

Equation (2.2) suggests that we define $h: V \rightarrow H$ by

$$
h(u)=\left(u^{\prime}-C u / 2\right) \int_{0}^{\eta} e^{-C r / 2} u(r) d r-e^{-C \eta / 2} u^{2} .
$$

Since $\|u\|_{\infty} \leq\|u\|_{1,2}$ for $u \in V$ there exists $c_{1}<\infty$ such that

$$
\begin{gathered}
\|h(u)\| \leq c_{1}\|u\|_{1,2}^{2} \quad \text { for all } u \in V, \\
\|h(u)-h(v)\| \leq c_{1}\|u-v\|_{1,2}\left(\|u\|_{1,2}+\|v\|_{1,2}\right) \quad \text { for all } u, v \in V .
\end{gathered}
$$

A couple of integrations by parts shows that for each $u \in V$ there exists $c_{u}<\infty$ such that

$$
|F(u, v)-\overline{F(v, u)}| \leq c_{u}\|v\| \quad \text { for all } v \in V .
$$

This implies that the domain of $(A-a)^{1 / 2}$ is equal to $V$ when $a<\operatorname{Re} \sigma(A)$ (see p. 253, p. 87 in [5]). Thus, all results in section 6.4 in [5] are applicable with $\alpha=1 / 2$. In particular,

Theorem 2.1. For any $u_{0} \in V$ there exists $\tau>0$ and a unique $\psi \in C([0, \tau), V)$ such that $\psi(0)=u_{0}$ and $\psi$ satisfies equation (2.4) on $(0, \tau)$. 
For small initial data, Theorem 6.4.11 in [5] implies global existence, stability and exponential decay of solutions of (2.4) when $\operatorname{Re} \sigma(A)>0$. This happens when $C>\sqrt{10}$ according to (2.9). According to numerical calculations below, this happens whenever $C>1$. We can improve (2.9) somewhat but we cannot match the numerical result.

Instability Theorem 6.4.12 in [5] adapted to this case states

Theorem 2.2. If Re $\lambda<0$ for some $\lambda \in \sigma(A)$, then there exists $\delta>0$ such that for each $\varepsilon>0$ there exists $\tau>0$ and $\psi \in C([0, \tau), V)$ which satisfies equation (2.4) on $(0, \tau)$ and is such that

$$
\|\psi(0)\|_{1,2}<\varepsilon \text { and }\|\psi(t)\|_{1,2}>\delta \text { for some } t>0 .
$$

Our instability assertion is a consequence of the existence of $\lambda \in \sigma(A)$ with $\operatorname{Re} \lambda<0$. We will prove this in the next section when $C$ is close to 1 and $C<1$. We will show this numerically when $0<C<1$ in Section 4 Theorem 6.4.12 in [5] also says that we can choose $\psi(0) \in \cap_{n=1}^{\infty} D\left(A^{n}\right)$, which implies regularity of $\psi$.

\section{Spectrum of A.}

Lemma 3.1. $\sigma(A) \backslash\left[C^{2} / 4, \infty\right)$ consists of isolated points of $\mathbb{C}$. Moreover, each point in $\sigma(A) \backslash\left[C^{2} / 4, \infty\right)$ is an eigenvalue of $A$ with (geometric) multiplicity 1 , and the corresponding spectral projection has a finite-dimensional range.

Proof. Let us write $A=T-B$ where $T u=-u^{\prime \prime}+C^{2} u / 4$, with $D(T)=D(A)$, and let $B$ represent the remaining terms in (2.8). Note that $\sigma(T)=\left[C^{2} / 4, \infty\right)$. For $\lambda \in \mathbb{C} \backslash\left[C^{2} / 4, \infty\right)$ we can write $(T-\lambda)^{-1}$ as an integral operator. Since $B$ contains an exponentially decaying factor we see that $B(T-\lambda)^{-1}$ is an integral operator with a square integrable kernel. Therefore $B(T-\lambda)^{-1}$ is a holomorphic family of compact operators for $\lambda \in \mathbb{C} \backslash\left[C^{2} / 4, \infty\right)$.

Let $S$ be the set of $\lambda \in \mathbb{C} \backslash\left[C^{2} / 4, \infty\right)$ for which 1 is an eigenvalue of $B(T-\lambda)^{-1}$. If $\lambda \in S$, then clearly $\lambda$ is an eigenvalue of $A$ and hence $\operatorname{Re} \lambda \geq\left(C^{2}-10\right) / 4$ by (2.9). Theorem 1.9 on p. 370 in [3] implies that the points of $S$ are isolated. If on the other hand $\lambda \notin S \cup\left[C^{2} / 4, \infty\right)$, then obviously

$$
(A-\lambda)^{-1}=(T-\lambda)^{-1}\left(1-B(T-\lambda)^{-1}\right)^{-1}
$$

and hence $S=\sigma(A) \backslash\left[C^{2} / 4, \infty\right)$.

If $u$ and $v$ are linearly independent eigenfunctions of $A$ corresponding to an eigenvalue $\lambda$, then a linear combination of $u$ and $v$ gives an eigenfunction $w \neq \equiv 0$ such that $w(0)=$ $w^{\prime}(0)=0$. Using (2.8) we see that $A w=\lambda w$ implies $w^{\prime \prime}(0)=0$ and since $A w=\lambda w$ can be rewritten as a 3 rd order ODE, we have a contradiction when $w \equiv 0$.

The spectral projection corresponding to $\lambda \in \sigma(A) \backslash\left[C^{2} / 4, \infty\right)$ is defined, as usual, by

$$
P_{\lambda}=\frac{1}{2 \pi i} \int_{\Gamma_{\lambda}}(z-A)^{-1} d z
$$

where $\Gamma_{\lambda}$ is a circle in the resolvent set of $A$ that contains $\lambda$ and no other part of $\sigma(A)$. Clearly,

$$
P_{\lambda}-0=\frac{1}{2 \pi i} \int_{\Gamma_{\lambda}}\left((z-A)^{-1}-(z-T)^{-1}\right) d z
$$


using (3.1),

$$
P_{\lambda}=\frac{1}{2 \pi i} \int_{\Gamma_{\lambda}}(z-T)^{-1} B(T-z)^{-1}\left(1-B(T-z)^{-1}\right)^{-1} d z .
$$

Since $B(T-z)^{-1}$ is compact, the integrand and hence $P_{\lambda}$ is compact, and since its range is closed, $P_{\lambda}$ has a finite-dimensional range.

The form (2.5) defines $A^{*}$, the adjoint of $A$, by

$$
F(u, v)=\left(u, A^{*} v\right) \quad \text { for } u \in V .
$$

It is easy to see that

$$
A^{*} v=-v^{\prime \prime}+\frac{C^{2}}{4} v+\frac{e^{-C \eta}}{C} v^{\prime}-\frac{5 e^{-C \eta}}{2} v-C e^{-C \eta / 2} \int_{\eta}^{\infty} e^{-C r / 2} v(r) d r
$$

for $v \in D\left(A^{*}\right)=W^{2}(0, \infty) \cap W_{0}^{1}(0, \infty)$.

Lemma 3.2. If $C=1$, then 0 is an isolated eigenvalue of $A$ with an

$$
\begin{gathered}
\text { eigenfunction of A: } \quad \psi_{0}(\eta)=\eta e^{-\eta / 2} \\
\text { eigenfunction of } A^{*}: \quad \phi_{0}(\eta)=\left(e^{-\eta / 2}-e^{-3 \eta / 2}\right) e^{-e^{-\eta}} \\
\left(\psi_{0}, \phi_{0}\right)=1-e^{-1}
\end{gathered}
$$

and the corresponding spectral projection has one dimensional range.

Proof. Using (2.8) it is easy to verify (3.4). $\phi_{0}$ given by (3.5) can be rewritten as

$$
\phi_{0}(\eta)=e^{-\eta / 2} \sum_{n=0}^{\infty}(n+1) \frac{\left(-e^{-\eta}\right)^{n}}{n !} .
$$

Using (3.7) in (3.3) gives $A^{*} \phi_{0}=0$. By using (3.7) it is also easy to verify (3.6). See also Figure 1 .

Let $P_{0}$ be the corresponding spectral projection given by (3.2), let $Y$ denote its range and let $n=\operatorname{dim} Y$. To prove that $n=1$ it is sufficient to prove

$$
A P_{0} u=0 \text { for all } u \in H
$$

because the zero eigenvalue has (geometric) multiplicity 1 by Lemma 3.1

Let $Q u \in \mathbb{C}^{n}$ give coordinates of $u \in Y$ with respect to a basis of $Y . \tilde{A}=Q A Q^{-1}$ is the matrix representing $A$ with respect to this basis. The fact that $A$ restricted to $Y$ has spectrum consisting of 0 only implies that the characteristic polynomial of $\tilde{A}$ is equal to $(-\lambda)^{n}$ and hence $\tilde{A}^{n}=0$ (Hamilton-Cayley Theorem).

Assuming $A P_{0} u_{1} \neq 0$ for some $u_{1}$ implies that there exists $y_{1}$ and $k \geq 1$ such that $\tilde{A}^{k} y_{1} \neq 0$ and $\tilde{A}^{k+1} y_{1}=0$. Thus, if $y_{3}=\tilde{A}^{k-1} y_{1}$, then

$$
y_{2}:=\tilde{A} y_{3} \neq 0, \quad \tilde{A} y_{2}=0 .
$$

Since $Q A Q^{-1} y_{2}=0$ we have to have that $A Q^{-1} y_{2}=0$, and hence $Q^{-1} y_{2}=c \psi_{0}$ for some c. $c \neq 0$ because $y_{2} \neq 0$ and hence we have a contradiction:

$$
0 \neq c\left(\psi_{0}, \phi_{0}\right)=\left(Q^{-1} y_{2}, \phi_{0}\right)=\left(A Q^{-1} y_{3}, \phi_{0}\right)=\left(Q^{-1} y_{3}, A^{*} \phi_{0}\right)=0 .
$$

Therefore (3.8) has to be true. 


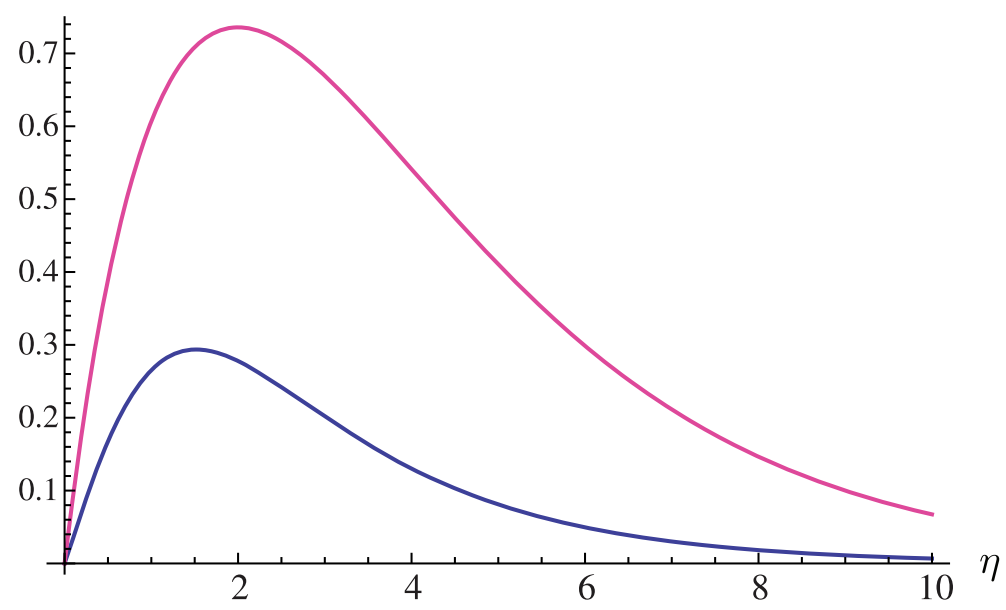

Fig. 1. Eigenfunctions $\psi_{0}(\eta)$ (above) and $\phi_{0}(\eta)$ given by (3.5).

THEOREM 3.3.

$$
\left.\frac{d \lambda}{d C}\right|_{C=1, \lambda=0}=\frac{2}{e-1}=1.163953
$$

and $A$ has a negative eigenvalue when $C \in(1-\varepsilon, 1)$ for some $\varepsilon>0$.

Proof. Let $\Gamma_{0}$ be a fixed circle around 0 that does not contain any part of $\sigma(A)$ except for 0 when $C=1$. For $C$ close to $1, A=A(C)$, define

$$
P(C)=\frac{1}{2 \pi i} \int_{\Gamma_{0}}(z-A)^{-1} d z .
$$

$P(1)$ is just the spectral projection $P_{0}$ corresponding to eigenvalue 0 , and it has one dimensional range by Lemma 3.2. The integrand in (3.10) is an analytic function of $C$ for $C$ close to 1 in a neighborhood of the range of the fixed $\Gamma_{0}$, so, $C \rightarrow P(C)$ is analytic near $C=1$ and hence the dimension of the range of $P(C)$ must remain $1 . P(C)$ is a spectral projection corresponding to some $\lambda(C)$ inside $\Gamma_{0}$ for $C$ close to 1 ; see 3 . Define

$$
\Psi(C)=P(C) \psi_{0}=\frac{1}{2 \pi i} \int_{\Gamma_{0}}(z-A)^{-1} \psi_{0} d z .
$$

Note that $\Psi(1)=\psi_{0}$ as given by (3.4) and $C \rightarrow \Psi(C)$ is analytic for $C$ close to 1 . Since $P(C)$ is a spectral projection, we have that

$$
A \Psi(C)=\lambda(C) \Psi(C) .
$$

This implies that $\lambda(1)=0$ and $C \rightarrow \lambda(C)$ is analytic for $C$ close to 1 . Differentiation of (3.11) with respect to $C$ at $C=1$ gives

$$
A^{\prime} \psi_{0}+A \Psi^{\prime}(1)=\lambda^{\prime}(1) \psi_{0}
$$

where

$$
2 A^{\prime} \psi_{0}=\psi_{0}+e^{-\eta}\left(2(1+\eta) \psi_{0}^{\prime}+3 \eta \psi_{0}\right)+\int_{0}^{\eta} e^{-(r+\eta) / 2}(r+\eta-2) \psi_{0}(r) d r
$$




$$
A^{\prime} \psi_{0}=\eta e^{-\eta / 2}+e^{-3 \eta / 2} .
$$

Taking a scalar product with $\phi_{0}$ on both sides of (3.12) gives

$$
\left.\frac{d \lambda}{d C}\right|_{C=1, \lambda=0}=\lambda^{\prime}(1)=\frac{\left(A^{\prime} \psi_{0}, \phi_{0}\right)}{\left(\psi_{0}, \phi_{0}\right)}
$$

Using (3.7) and (3.13) gives

$$
\left(A^{\prime} \psi_{0}, \phi_{0}\right)=2 e^{-1}
$$

This and (3.6) imply (3.9).

4. Numerical computation of the spectrum. Pick a truncation distance $L>0$, $n>1$ and let $h=L /(n+1)$. A straightforward discretization of $\lambda u=A u$ gives for $1 \leq i \leq n$

$$
\begin{gathered}
\lambda u_{i}=-\frac{u_{i+1}+u_{i-1}-2 u_{i}}{h^{2}}+\frac{C^{2}}{4} u_{i}-\frac{e^{-C i h}}{C} \frac{u_{i+1}-u_{i-1}}{2 h}-\frac{3 e^{-C i h}}{2} u_{i} \\
-C h\left(\frac{1}{2} e^{-C i h} u_{i}+\sum_{j=1}^{n-1} e^{-C(i+j) h / 2} u_{j}\right)
\end{gathered}
$$

where $u_{i}$ is an approximation of $u(i h) . \quad u_{0}=0$ due to the boundary condition and $u_{n+1}=0$ due to the truncation.

Use of central differences and the trapezoid formula in (4.1) results in very well behaved errors. Error terms in approximations of $\lambda \in \sigma(A)$ are proportional to even powers of $h$ and hence we can eliminate them one by one (Richardson extrapolation). We typically do this two times - ending with errors of order $h^{6}$.

The leading eigenvalue, when away from the continuous spectrum, does not depend much on the truncation length $L$. For example, one obtains the same 9 significant digits when $50 \leq L \leq 400$ for a reference value

$$
\text { leading eigenvalue } \lambda=-0.244314795 \text { when } C=0.8 \text {. }
$$

Other eigenvalues of (4.1) seem to be approximations of the continuous spectrum and do depend on $L$. The eigenvalue approximating the beginning of the continuous spectrum is approximately $C^{2} / 4+(\pi / L)^{2}$ - as one would expect from $T u=-u^{\prime \prime}+C^{2} u / 4$ on $L^{2}(0, L)$.

The leading eigenvalue, as a function of $C$, is represented by the lower curve on Figure 2. Note that it is negative for $C \in(0,1)$, indicating instability, and positive for $C>1$. The bottom of the continuous spectrum $\left(C^{2} / 4\right)$ is represented by the upper curve on Figure 2, The leading eigenvalue gets absorbed into the continuous spectrum near $C=1.7$. The vertical cross sections, like the one shown at $C=0.8$, show the spectrum of $A$.

To see how the predicted crossover rate (3.9) compares to the numerical estimate, we calculated

$$
\begin{gathered}
\text { when } C=1.01, \quad \lambda=0.011613 \\
\text { when } C=0.99, \quad \lambda=-0.011666 .
\end{gathered}
$$




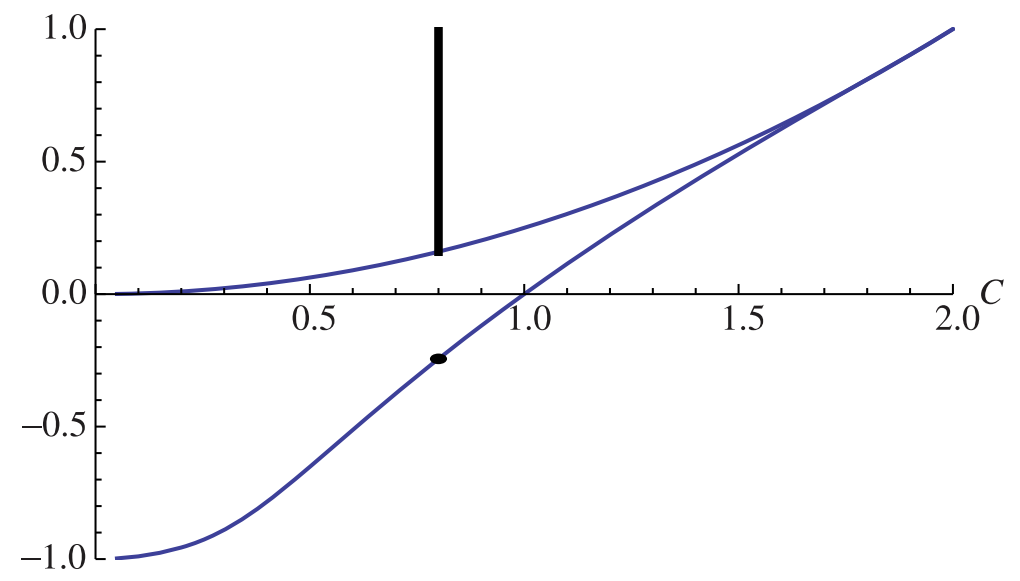

FIG. 2. The bottom curve shows the leading eigenvalue as a function of $C$. The top curve shows the bottom point $\left(C^{2} / 4\right)$ of the continuous spectrum.

Hence the numerical approximation of the rate of change of eigenvalue $\lambda=0$ is

$$
(0.011613+0.011666) / .02=1.16395,
$$

which is very close to the predicted value given by equation (3.9).

5. Notes. What would happen if we look for perturbations of the stationary solution that are in the form

$$
f(\eta, \tau)=C+\frac{1}{C} e^{-C \eta}+\int_{0}^{\eta} e^{-\omega r} \psi(r, \tau) d r
$$

where $\omega \in[0, C)$ ? In (2.1) we assumed $\omega=C / 2$. We can proceed as in Section 2 and let $A_{\omega}$ be the new equivalent of $A$ given by (2.8). Let us split $A_{\omega}$ as in Lemma 3.1

$$
A_{\omega}=T_{\omega}-B_{\omega}
$$

where

$$
\begin{gathered}
T_{\omega} u=-u^{\prime \prime}+(2 \omega-C) u^{\prime}+\omega(C-\omega) u \\
B_{\omega} u=\frac{e^{-C \eta}}{C} u^{\prime}+(2-\omega / C) e^{-C \eta} u+C e^{-(C-\omega) \eta} \int_{0}^{\eta} e^{-\omega r} u(r) d r
\end{gathered}
$$

and $D\left(A_{\omega}\right)=D\left(T_{\omega}\right)=D\left(B_{\omega}\right)=W^{2}(0, \infty) \cap W_{0}^{1}(0, \infty)$. As before, $B_{\omega}$ is a relative compact perturbation of $T_{\omega}$, and the resolvent set of $T_{\omega}$ can contain only isolated eigenvalues of $A_{\omega}$. However, the resolvent set of $T_{\omega}$ is very different:

$$
\sigma\left(T_{\omega}\right)=\left\{\lambda \in \mathbb{C} \mid(\operatorname{Im} \lambda)^{2} \leq(2 \omega-C)^{2}(\operatorname{Re} \lambda-\omega(C-\omega)), \operatorname{Re} \lambda \geq \omega(C-\omega)\right\} .
$$

The continuous spectrum of $T_{\omega}$ is pushed furthest away from 0 when $\omega=C / 2$ and, when $\omega=0,0$ is a part of the continuous spectrum of $T_{\omega}$. On the other hand, the leading eigenvalue does not depend on $\omega$. When $C=1$ it is easy to see that 0 is an eigenvalue of $A_{\omega}$ for all $\omega \in[0,1)$ with the corresponding eigenfunction $\eta e^{(\omega-1) \eta}$. The above argument showing that the 0 eigenvalue crosses an imaginary axis breaks down 
when $\omega=0$ because 0 is also in the continuous spectrum of $T_{0}$. However, we can obtain an eigenfunction with a negative eigenvalue in the case $\omega=0$ when $C<1$ and $C$ is close to 1 by simply multiplying the unstable eigenfunction provided in Theorem 3.3 by $e^{-\eta / 2}$. Therefore the assumption of Theorem 2.2 is also satisfied in the case $\omega=0$ when $C<1$ and $C$ is close to 1 .

\section{REFERENCES}

[1] Daniel Henry, Geometric theory of semilinear parabolic equations, Lecture Notes in Mathematics, vol. 840, Springer-Verlag, Berlin, 1981. MR610244 (83j:35084)

[2] Daniel D. Joseph, Stability of fluid motions. I, Springer-Verlag, Berlin, 1976. Springer Tracts in Natural Philosophy, Vol. 27. MR0449147 (56 \#7452)

[3] T. Kato, Perturbation Theory for Linear Operators, 2nd edition, Springer, New York, 1980.

[4] Milan Miklavčič, Nonlinear stability of asymptotic suction, Trans. Amer. Math. Soc. 281 (1984), no. 1, 215-231, DOI 10.2307/1999531. MR719667 (84m:35098)

[5] Milan Miklavčič, Applied functional analysis and partial differential equations, World Scientific Publishing Co. Inc., River Edge, NJ, 1998. MR.1784426 (2001k:47001)

[6] M. Miklavčič and C. Y. Wang, Viscous flow due to a shrinking sheet, Quart. Appl. Math. 64 (2006), no. 2, 283-290. MR2243864 (2007c:76021)

[7] C. Y. Wang, Q. Du, M. Miklavčič, and C. C. Chang, Impulsive stretching of a surface in a viscous fluid, SIAM J. Appl. Math. 57 (1997), no. 1, 1-14, DOI 10.1137/S0036139995282050. MR1429374 (97k:76032) 